\title{
Heavy flavors on CT14
}

Tie-Jiun Hou ${ }^{* a}$, Sayipjamal Dulat ${ }^{b, c}$, Jun Gao ${ }^{d}$, Marco Guzzi ${ }^{e}$, Joey Huston $c$, Pavel Nadolsky $^{a}$, Jon Pumplin ${ }^{c}$, Carl Schmidt $^{c}$, Daniel Stump ${ }^{c}$, C.-P. Yuan ${ }^{c}$

${ }^{a}$ Department of Physics, Southern Methodist University, Dallas, TX 75275-0181, U.S.A.

${ }^{b}$ School of Physics Science and Technology, Xinjiang University, Urumqi, Xinjiang 830046

China

${ }^{c}$ Department of Physics and Astronomy, Michigan State University, East Lansing, MI 48824

U.S.A.

${ }^{d}$ High Energy Physics Division, Argonne National Laboratory, Argonne, Illinois 60439, U.S.A.

${ }^{e}$ School of Physics \& Astronomy, University of Manchester, Manchester M13 9PL, United

Kingdom

E-mail: tiejiunh@mail.smu.edu

We report on the recent study of possible intrinsic charm component of proton Parton Distribution Function base on the CT14 Next-to-Next-to-Leading Order. We also report on the study of uncertainties of Higgs boson and $t \bar{t}$ production at the LHC center-of-mass energy $13 \mathrm{TeV}$ from PDF associate with the strong coupling $\alpha_{s}\left(M_{z}\right)$. Uncertainties that are estimated by the Hessian method and the Lagrange Multiplier method are in a good agreement.

XXIII International Workshop on Deep-Inelastic Scattering

27 April - May 12015

Dallas, Texas

\footnotetext{
*Speaker.
} 


\section{Updataed study on Intrinsic Charm from CT14 global analysis}

The CT14 next-to-next-to-leading order(NNLO) parton distribution function(PDF) is the first global analysis which include the data from LHC in the CTEQ-TEA(CT) series [1]. The CT14 NNLO(referred to as CT14 here) also include the updated data from the Tevatron and from HERA experiments. Base on the CT10 data set [2], the CT14 update the HERA $F_{L}$ and $F_{2}^{c}$ data [3] and include the LHC RUN I data at $7 \mathrm{TeV}$. The included LHC data contain the W/Z production from ATLAS [4] and LHCb [5]; the W-lepton charge asymmetry from ATLAS [4], CMS [6, 7] and LHCb [5]; and the inclusive jet data from ATLAS [8] and CMS [9]. The W-electron rapidity asymmetry data from D0 with larger intgrated lumiosity [10] is updated. More flexible parametrization by the implementation of the Bernstein polynomials is applied. The number of PDF parameters used in the CT14 global analysis increased to 28, compared to 25 in CT10 NNLO. There are a total of 2947 data points included from 33 experiments, producing $\chi^{2}=3250$ at the best fit with $\chi^{2} / N_{p t}=1.10$.

In the global analysis the charm quark is generally considered as heavy quark and generated radiatively through evolution. This assumption base on the lack of clearly identifiable experimental constraints on the degree of freedom of heavy quarks in existing data. However, the charm quark is special with its mass lies in between the soft and hard energy scale, and many nonperturbative models predict the existence of the intrinsic charm component of nucleon at energy scale of charm quark.

The intrinsic charm was studied base on the CTEQ 6.5 with next-to-leading-

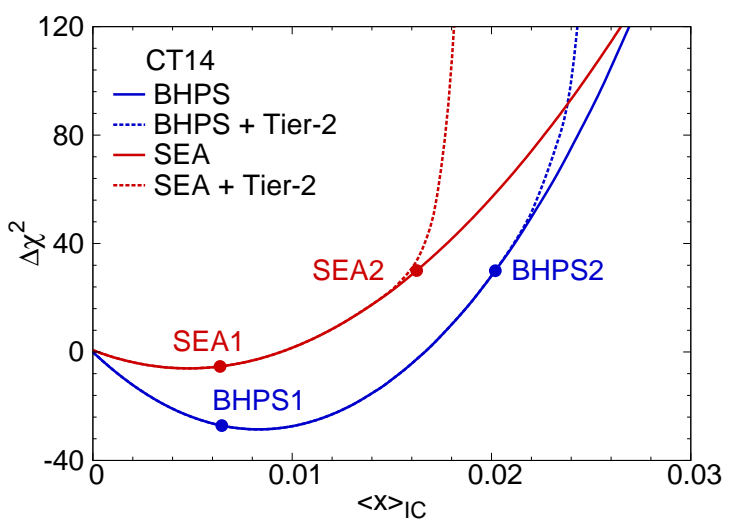

Figure 1: The $\Delta \chi^{2}$ versus the momentum fraction of charm $\langle x\rangle_{I C}$. order (NLO) approximation for perturbative

QCD [11]. The study concluded no evidence either for or against IC up to $\langle x\rangle_{I C} \sim 0.01$. The updated study was done base on the CT10 NNLO [12] with inclusion of the combined H1/ZEUS data for deep-inelastic scattering [19] and inclusive charm production at HERA [14]. Using a charm pole mass of $m_{c}=1.3 \mathrm{GeV}$, which is compatable with the study of charm quark mass to be $1.15_{-0.12}^{+0.18} \mathrm{GeV}$ from global analysis [15], two models was considered: the valence-like Brodsky, Hoyer, Peterson, and Sakai(BHPS) model and the sea-like "SEA" model. Both of the models have an single parameter, which can be traded by the momentum fraction of the intrinsic charm $\langle x\rangle_{I C}$. The updated study concluded the upper limits on $\langle x\rangle_{I C}$ at $90 \%$ C.L. to be 0.025 for BHPS model and 0.015 for SEA model.

With the includsion of the data from LHC RUN I, updated study of intrinsic charm base on CT14 NNLO is done by appling the Lagrange Multiplier method. In fig. 1 we plot the $\Delta \chi^{2}$ versus the momentum fraction of charm $\langle x\rangle_{I C}$ for the two different models, where the $\Delta \chi^{2}=100$ is considered as $90 \%$ C.L. in the CT14 global analysis. The Tier-2 penalty shows in the figure is the additional contribution to the $\chi^{2}$ to ensure the goodness of all individual fits in the global analy- 

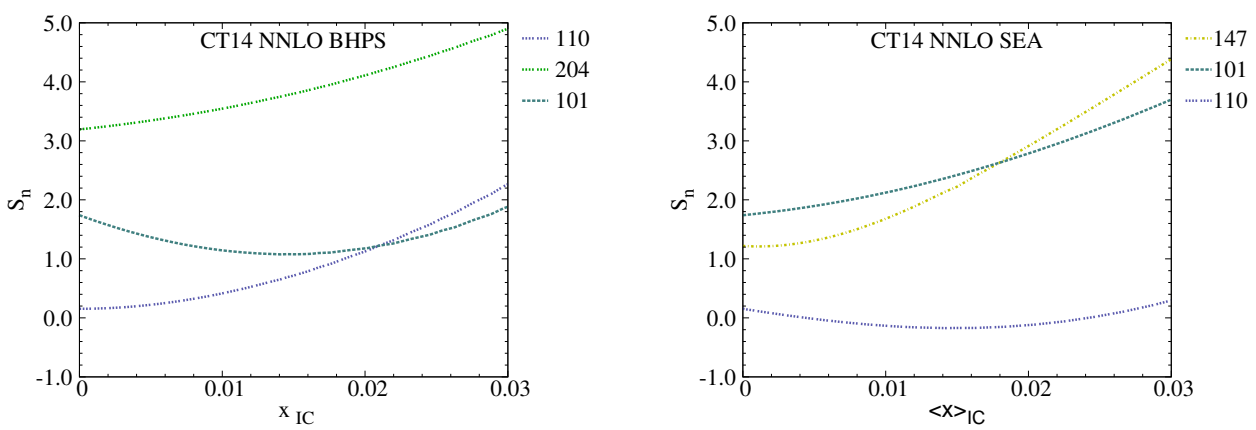

Figure 2: The spartyness $S_{n}$ versus the charm momentum fraction $\langle x\rangle_{I C}$.

sis [12]. As expected, because the new included LHC data are not sensitive to the intrinsic charm, it shows no significant difference from the last study base on CT10 NNLO. In fig. 2 we present the sensitivities of data by ploting the effective Gaussian variable "spartyness" $S_{n}$ [12] versus the momentum fraction of the intrinsic charm $\langle x\rangle_{I C}$. For the BHPS model, the CCFR $F_{2}^{p}$ measurement(110) [16] and E866 Drell-Yan process(204) [17] prevent large component of the valence-like intrinsic charm in the proton. For the SEA model, the combined HERA charm production(147) [14] and the BCDMS $F_{2}^{p}$ measurement(101) [18] against the large sea-like intrinsic charm.

\section{Prediction in Higgs and $t \bar{t}$ production}

One of the dominant on-going plan of the LHC is the search of physics beyond Stand Model. The RUN II of the LHC has higher center-of-mass energy and larger integrated luminosity, and thus is more potential on the discovery of new physics. In hadron collider, the Higgs boson production through gluon fusion would be very important for determining the Higgs profile. As the statistic error to be reduced by the large integrated luminosity in LHC RUN II, and higher order theoretical calculation reduce the scale dependence, the uncertainty from PDF and strong coupling constant $\alpha_{s}$ would be the dominant uncertainty.

The standard method for deteriming the

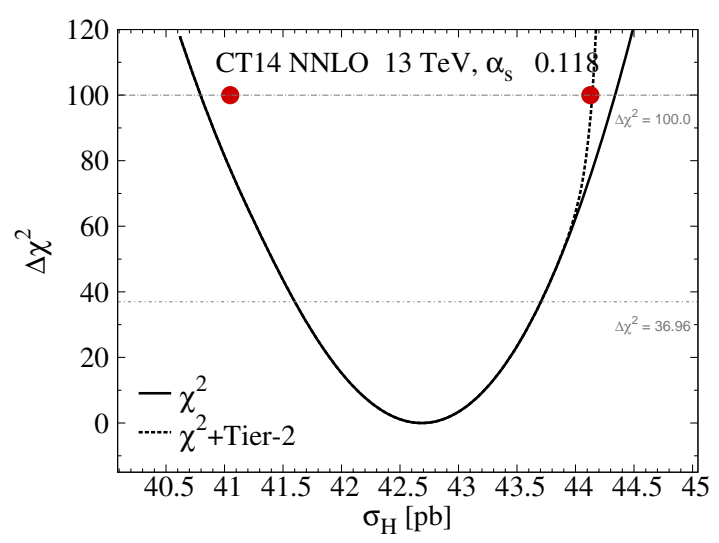

Figure 3: $\chi^{2}$ versus $\sigma_{H}$ with $\alpha_{s}=0.118$ at $13 \mathrm{TeV}$. The curves shows the LM scan without(solid) and with(dashed) the Tier-2 penalty. The Hessian uncertainty at $90 \%$ C.L. is presented by red dots. PDF uncertainty is the Hessian method. The uncertainty of the PDFs is estimated by the Hessian method in the CT14 global analysis. The Hessian method has the virtue of convenience for estimating the uncertainty of PDF and observables by the eigenvector sets. By using the eigenvector sets, the symmetric and asymmetic uncertainties of the physical observables and PDFs can be worked out by appling the master formula. It is also a powerful tool for considering the correlation between observables. In practice, the Hessian method 

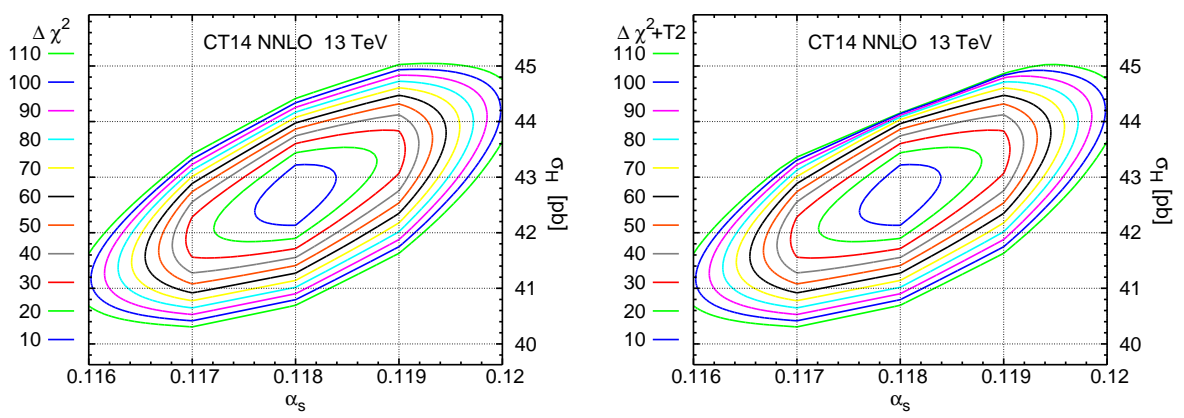

Figure 4: Contour of $\Delta \chi^{2}$ and $\Delta \chi^{2}+$ Tier-2 penalty in the $\left(\alpha_{s}, \sigma_{H}\right)$ plane obtained by the LM method.

assume a quadratic $\chi^{2}$ potential and a linear dependency between the observable and the PDF fitting parameters around the neighborhood of the minimum. The Lagrange Multipliers(LM) method is the alternative way for the uncertainty estimation. The LM method does not depend on the shape of the $\chi^{2}$ nor the linearity to the fitting parameters. The inconvenience of the Lagrange multiplier method is the need of many fittings for obtaining the uncertainty of one particular observable. The LM method would then be a tool for testing the reliability of the Hessian method.

In fig. 3, we show the uncertainty of Higgs boson production with fixed $\alpha_{s}=0.118$ at $13 \mathrm{TeV}$ from Hessian method and LM method. The solid and dashed curves are calculated by the LM method without and with Tier-2 pantly respectively. The red circle is the uncertainty calculated by Hessian method with Tier-2 penalty at the $90 \%$ C.L.. It shows that, the uncertainty obtained by Hessian method is in a good agreement with the one by LM method. The differences between them are considerably smaller than the errors themselves.

The uncertainty of $\mathrm{PDF}+\alpha_{s}$ in Hessian method is calculated by including two additional error PDFs on $\alpha_{s}$. The PDF4LHC [21] choose the $\alpha_{s}\left(M_{z}\right)=0.118 \pm 0.002$ at $90 \%$ C.L.. The two additoinal error PDFs in Hessian method at $90 \%$ C.L. correspond to $\alpha_{s}\left(M_{z}\right)=0.116$ and $\alpha_{s}\left(M_{z}\right)=0.120$. In the LM method, the uncertainty of PDF $+\alpha_{s}$ can be worked out by the scan in the $\alpha_{s}-\sigma_{H}$ plane [22]. In fig.4 we show the contour plot with and without the contribution from Tier2 penalty in the $\left(\alpha_{s}, \sigma_{H}\right)$ plane. The deviation from pure ellipses of the contours indicate the non-quadratic shape in the $\chi^{2}$ potential. It worth to notice that, the contour of $90 \%$ C.L., which correspod to the ellipse of $\Delta \chi^{2}=100$, receive contribution from the

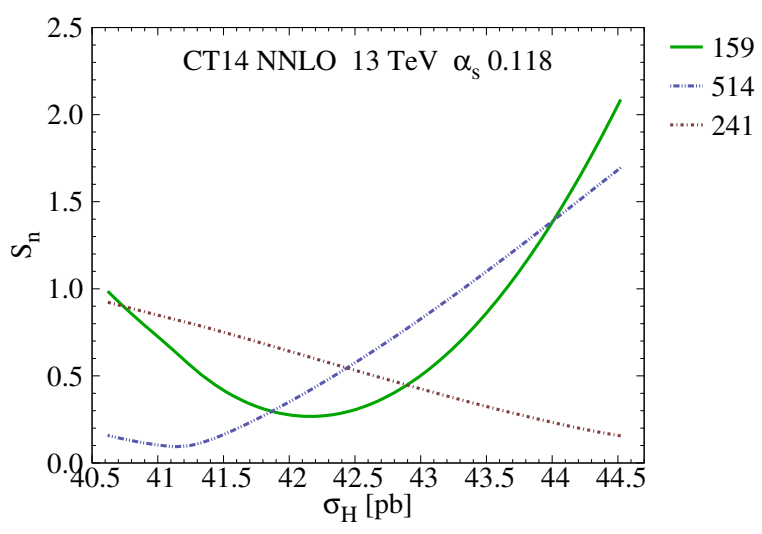

Figure 5: The effective Gaussian variable spartyness versus Higgs cross section. The sensitivity of data of HERA1 Combined NC and CC DIS(159) [19], D0 RUN II inclusive jet(514) [20] and LHCb $7 \mathrm{TeV}$ charge lepton asymmetry(241) [5] are presented.

Tier-2 penalty on the upper part of the contour. The uncertainties calcualted by the Hessian method and the LM method are still in good agreement as summarized in table. 1. The Hessian 68\% C.L. uncertainty can be calculated by the $90 \%$ C.L. uncertainty devided by 1.645 . The LM method is 
also a powerful tool for observing the sensitivity of data for a particular obervable. In fig.5, we show the sensitivity of data versus Higgs production with fixed $\alpha_{s}=0.118$ at $13 \mathrm{TeV}$. It shows that, the combined HERA RUN I data have tension when the Higgs production goes too large and contribute to the Tier-2 penalty.

\begin{tabular}{l|c}
\hline$g g \rightarrow H(\mathrm{pb}), \mathrm{PDF}$ unc., $\alpha_{s}=0.118$ & $13 \mathrm{TeV}$ \\
\hline 90\% C.L. $(H e s s i a n)$ & $42.7+3.3 \%-3.9 \%$ \\
\hline $90 \%$ C.L. $(\mathrm{LM})$ & $42.7+3.4 \%-4.4 \%$ \\
\hline \hline$g g \rightarrow H(\mathrm{pb}), \mathrm{PDF}+\alpha_{s}$ & $13 \mathrm{TeV}$ \\
\hline 90\% C.L. $(\mathrm{Hessian})$ & $42.7+4.9 \%-5.3 \%$ \\
\hline 90\% C.L. $(\mathrm{LM})$ & $42.7+5.0 \%-5.3 \%$ \\
\hline
\end{tabular}

Table 1: PDF and PDF $+\alpha_{s}$ uncertainties of gluon fusion Higgs boson production $\sigma_{H}(g g \rightarrow H)$ at $13 \mathrm{TeV}$ computed by the Hessian method and the LM method with Tier-2 penalty included.
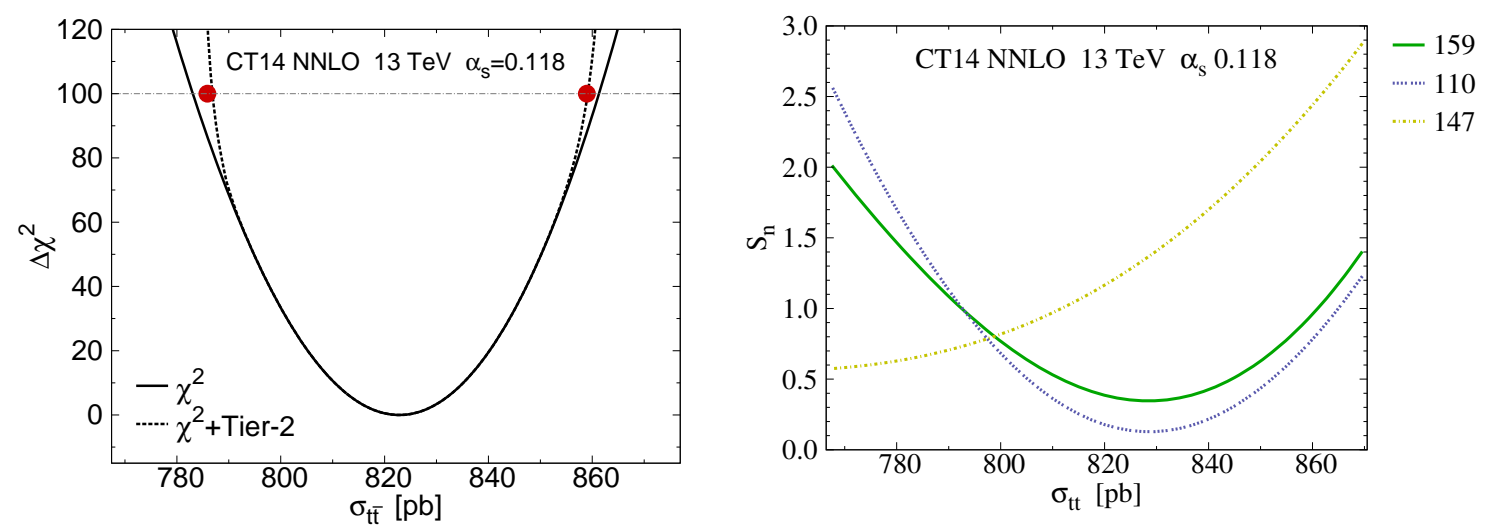

Figure 6: The $\Delta \chi^{2}$ and spartyness versus the $t \bar{t}$ production with $\alpha_{S}\left(M_{z}\right)=0.118$ at $13 \mathrm{TeV}$. In the right penal, the sensitivities of the HERA1 Combined NC and CC DIS(159), the CCFR $F_{2}^{p}$ measurement(110) and the combined HERA charm production(147) are presented.

The $t \bar{t}$ production is also an important channel in LHC as the standard model measurement and the background to new physics. We repeat the same analysis for the $t \bar{t}$ production to checking the agreement of the Hessian and LM uncertainties. In fig.6, we present the comparison of $90 \%$ C.L. uncertainties by the Hessian and LM methods for $t \bar{t}$ production with $\alpha_{S}\left(M_{z}\right)=0.118$ at 13 $\mathrm{TeV}$. Again, the Hessian and LM uncertainties are in good agreement with the inclusion of Tier-2 penalty. The figure of sesitivity of data show in the right penal explains the tension from the combined HERA charm production on the larger $t \bar{t}$ production and from the CCFR $F_{2}^{p}$ measurement and combined HERA DIS data on the lower production. The comparison of PDF and PDF $+\alpha_{s}$ uncertainties between the Hessian and LM methods are also performed and summarized in table.2. The difference between the uncertainties from two methods is small as compare to the uncertainties themselves. We, therefore, verify the reliability of the Hessian method for $t \bar{t}$ production at LHC. 


\begin{tabular}{l|c}
\hline$p p \rightarrow t \bar{t}(\mathrm{pb}), \mathrm{PDF}$ unc., $\alpha_{s}=0.118$ & $13 \mathrm{TeV}$ \\
\hline 90\% C.L. $($ Hessian$)$ & $820+4.4 \%-4.5 \%$ \\
\hline 90\% C.L. $(\mathrm{LM})$ & $820+4.4 \%-4.4 \%$ \\
\hline \hline$p p \rightarrow t \bar{t}(\mathrm{pb})$, PDF $+\alpha_{s}$ & $13 \mathrm{TeV}$ \\
\hline 90\% C.L. $($ Hessian) & $820+5.8 \%-5.7 \%$ \\
\hline 90\% C.L. $(\mathrm{LM})$ & $820+6.0 \%-5.9 \%$ \\
\hline
\end{tabular}

Table 2: The $t \bar{t}$ cross sections given in pb are evaluated at LHC center of mass energies of $13 \mathrm{TeV}$.

\section{References}

[1] S. Dulat et al., arXiv:1506.07443 [hep-ph].

[2] J. Gao, M. Guzzi, J. Huston, H. -L. Lai, Z. Li, P. Nadolsky, J. Pumplin and D. Stump et al., arXiv:1302.6246 [hep-ph].

[3] F. Aaron et al. (H1 Collaboration), Eur.Phys.J. C71, 1579 (2011), 1012.4355.

[4] G. Aad et. al. (ATLAS Collaboration), Phys.Rev. D85, 072004 (2012), 1109.5141.

[5] R. Aaij et. al. (LHCb Collaboration), JHEP 1206, 058 (2012), 1204.1620.

[6] S. Chatrchyan et. al. (CMS Collaboration), Phys.Rev. D90, 032004 (2014), 1312.6283.

[7] S. Chatrchyan et. al. (CMS Collaboration), Phys.Rev.Lett. 109, 111806 (2012), 1206.2598.

[8] G. Aad et. al. (ATLAS Collaboration), Phys.Rev. D86, 014022 (2012), 1112.6297.

[9] S. Chatrchyan et al. (CMS Collaboration), Phys.Rev. D87, 112002 (2013), 1212.6660.

[10] V. M. Abazov et al. (DØ Collaboration), Phys. Rev. D91, no. 3, 032007 (2015) [erratum: Phys. Rev. D91, no. 7, 079901 (2015)] [arXiv:1412.2862 [hep-ex]].

[11] J. Pumplin, H. L. Lai and W. K. Tung, Phys. Rev. D 75, 054029 (2007) [hep-ph/0701220].

[12] S. Dulat, T. J. Hou, J. Gao, J. Huston, J. Pumplin, C. Schmidt, D. Stump and C.-P. Yuan, Phys. Rev. D 89, no. 7, 073004 (2014) [arXiv:1309.0025 [hep-ph]].

[13] F. D. Aaron et al. [H1 and ZEUS Collaborations], JHEP 1001, 109 (2010) [arXiv:0911.0884 [hep-ex]].

[14] H. Abramowicz et al. [H1 and ZEUS Collaborations], Eur. Phys. J. C 73, no. 2, 2311 (2013) [arXiv:1211.1182 [hep-ex]].

[15] J. Gao, M. Guzzi and P. M. Nadolsky, Eur. Phys. J. C 73, no. 8, 2541 (2013) [arXiv:1304.3494 [hep-ph]].

[16] U. K. Yang et al. [CCFR/NuTeV Collaboration], Phys. Rev. Lett. 86, 2742 (2001) [hep-ex/0009041].

[17] J. Webb et. al. (NuSea Collaboration) (2003), hep-ex/0302019.

[18] A. C. Benvenuti et al. [BCDMS Collaboration], Phys. Lett. B 223, 485 (1989).

[19] F. Aaron et. al. (H1 and ZEUS Collaboration), JHEP 1001, 109 (2010), 0911.0884.

[20] V. Abazov et. al. (DØ Collaboration), Phys.Rev.Lett. 101, 062001 (2008), 0802.2400.

[21] S. Alekhin et al., arXiv:1101.0536 [hep-ph].

[22] S. Dulat, T. -J. Hou, J. Gao, J. Huston, P. Nadolsky, J. Pumplin, C. Schmidt and D. Stump and C. -P. Yuan, arXiv:1310.7601 [hep-ph]. 